\title{
THE POTENTIAL OF 360-DEGREE VIDEOS FOR TEACHING, LEARNING AND RESEARCH
}

\author{
J. Reyna \\ University of Technology Sydney (AUSTRALIA) \\ jorge.reyna@uts.edu.au
}

\begin{abstract}
Cutting-edge digital technologies could impact teaching, learning and research by providing more efficient, flexible and immerse experiences. In the early $90 \mathrm{~s}$, Apple developed QuickTime Virtual Reality (QTVR), and it can be considered the precursor of 360-degree videos. QuickTime VR technology used a series of pictures and stitched them together cylindrically (images wrapped around the viewer) using a QuickTime movie file. Users were able to scroll up and down, right and left, zoom in and out and even click links that contained audio or pop-up windows. In the late 90s, applications such as PanoViewer were developed using Flash that has similar functionality. With the mobile phone (2007) and tablet revolution (2010), these applications became redundant, and mobile applications started to offer VR experiences. Regrettably; it never has a massive uptake for education nor for the general public. Twenty years later, the offer of 360-degree video cameras started due to YouTube support for publishing and viewing the 360-degree videos (2015). Currently, there are more than twenty 360 -degree video camera brands on the market. The development of a vast offer of action cams may inspire this trend. This conceptual paper presents a critical discussion of how this new technology could potentially be embedded in teaching, learning and research, and what are their advantages and limitations.
\end{abstract}

Keywords: VR, 360-degree videos, 360 videos, virtual reality, action cams in education.

\section{Cite as:}

Reyna, J (2018). The Potential of 360-Degree Videos for Teaching, Learning and Research. Rethinking Learning in a Connected Age. The 12th annual International Technology, Education and Development Conference, INTED2018, Valencia (Spain), March 5th-7th.

Full paper has been accepted and it will be available online at INTED 2018 Proceedings. 